Résumés des conférences et travaux

\title{
Démotique
}

\section{Initiation à la littérature sapientiale égyptienne}

\section{Damien Agut-Labordère}

\section{(2) OpenEdition Journals}

Édition électronique

URL : https://journals.openedition.org/ashp/140

DOI : 10.4000/ashp.140

ISSN : 1969-6310

Éditeur

Publications de l'École Pratique des Hautes Études

\section{Édition imprimée}

Date de publication : 1 octobre 2008

Pagination : 5-7

ISSN : 0766-0677

\section{Référence électronique}

Damien Agut-Labordère, «Initiation à la littérature sapientiale égyptienne », Annuaire de l'École pratique des hautes études (EPHE), Section des sciences historiques et philologiques [En ligne], 139 | 2008, mis en ligne le 09 décembre 2008, consulté le 12 juillet 2021. URL : http://journals.openedition.org/ashp/140 ; DOI : https://doi.org/10.4000/ashp.140 


\title{
INITIATION À LA LITTÉRATURE SAPIENTIALE ÉGYPTIENNE
}

\author{
Chargé de conférences : M. Damien Agut-Labordère
}

Programme de l'année 2006-2007 : I. Étude des recueils de prohibitions. — II. Lexicographie. - III. Littérature et histoire économique et sociale.

Durant l'année 2006-2007, nous avons successivement proposé l'étude d'un genre particulier de sagesse égyptienne, les recueils de prohibitions, puis, une série de séances consacrées à la lexicographie et, enfin, un examen de la manière dont le monde paysan égyptien apparaissait dans les sagesses tardives.

\section{I. Étude des recueils de prohibitions}

Un recueil de prohibitions est une sagesse formée uniquement d'interdictions. On connaît ce type de texte par plusieurs ostraca fragmentaires provenant de Deir elMédina dont Frederik Hagen a proposé récemment une édition complète ${ }^{1}$. Les proverbes sont presque toujours composés en deux temps. La première partie est constituée d'une forme verbale au vétitif. La seconde présente un grand nombre de variations qui permettent de compléter le sens de l'interdiction. Après avoir repris les passages essentiels de ce texte, afin de souligner notamment certains aspects grammaticaux, nous avons montré que ce genre de sagesse n'était pas propre au Nouvel Empire. En effet, nous le retrouvons en démotique ce qui contribue à souligner la forte continuité qui existe entre les sagesses « classiques » et les sagesses démotiques. Ainsi, le $P$. Louvre N 2414, provenant des archives du reclus Ptolémaios qui vivait sous les règnes de Ptolémée VII et VIII, recèle, au recto, une sagesse de ce type. De la même manière, une longue séquence du $P$. Insinger ( $P$. Insinger 10. 10-23 - 11. 12) contient une suite de monostiches au vétitif.

\section{Lexicographie}

Nous avons ensuite entamé la partie lexicographique de notre programme par l'examen des termes employés dans les sagesses démotiques pour désigner l'avidité : $\check{s} g g$, 'w-n-hi.t, 'f' et $s g n$. Un examen précis des différentes occurrences nous a permis d'affiner la signification de chacun de ces termes : šgg désigne le fait d'être sous l'emprise d'une passion sans que celle-ci soit précisée, ' $w$ - $n$ - $\underline{h} . \underline{t}$ renvoie à la concupiscence, ' $f$ ' exprime la cupidité, enfin sgn est un mot plus ancien (dont l'emploi remonte au moins au Moyen Empire) et qui désigne soit la cupidité, soit la concupiscence.

Le mot wsthn, « marcher sans rencontrer d'obstacles » [Wb. I 367], est un terme à l'étymologie problématique dont l'usage est assez courant dans les sagesses tardives.

1. F. Hagen, « The Prohibitions : A New Kingdom Didactic Text », JEA, 97 (2005), p. 125-155. 
Dans ces textes, son sens s'enrichit considérablement. À travers l'étude de l'ensemble des occurrences, nous avons vu qu'il peut alors revêtir trois acceptions : 1) « être libéré d'une obligation »;2) « être désinvolte »; 3) « être généreux ».

Le mot hny.t [Wb. III, 102, 16-17] désigne à l'origine de l' « encens », une « chose », une « offrande » ou le « service de l'offrande lui-même » ${ }^{1}$. En démotique, il renvoie à une « affaire » au sens commercial du terme :

P. Insinger 26. 19-20

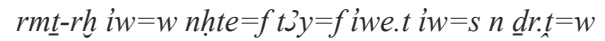

hr ir hrw $=$ f h hn hny.t iwe.t iw mn ' $n \underline{h}$

« Un sage en qui l'on a confiance, sa caution est dans ses mains.

(Car), en matière d'affaires, sa parole sert de gage sans qu'il ait besoin de prêter serment. »

Il se peut aussi qu'il serve à nommer, de manière plus précise, un « investissement » comme le montrent au moins deux exemples tirés du P. Insinger.

P. Insinger 19. $23-20.1$

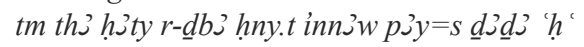

«Ne regrette pas un investissement lorsque son capital ${ }^{2}$ demeure intact. »

P. Insinger $3.24-4.1$

tm fy w'ไ irm hny.t iw pjy=s šy h̆'r

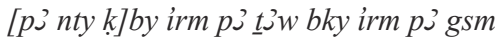

« N'ajoute pas d'investissement si le sort s'acharne (contre une affaire).

(Car) celui qui lutte contre le vent, il chavirera avec la tempête. »

\section{Littérature et histoire économique et sociale}

Nous avons enfin conduit une étude du thème du paysan et des pratiques agricoles dans les sagesses égyptiennes. Dans ce cadre, nous avons retraduit entièrement l'«éloge du paysan » que l'on trouve dans le P. Brookyn 47.218. 135. À la lumière de ce travail, nous avons vu que ce texte a moins vocation à louer les mérites du simple paysan que ceux du propriétaire terrien qui tire du profit des travaux de ses fermiers (iḩwty.w). Ce thème peut être rapproché de l'inscription $51 \mathrm{du}$ tombeau de Pétosi-

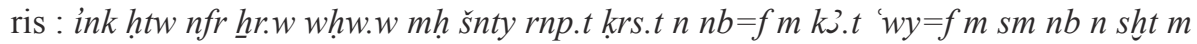
iy ’ht : «C'est moi, le bon fermier qui porte les grains, qui remplit le grenier (même) la mauvaise année pour son maître grâce au travail de ses bras, avec toutes sortes de plantes des champs quand vient la saison-akhet. »

Signalons aussi un rapprochement intéressant entre ce texte et un document littéraire égyptien. Ainsi, le proverbe cité en P. Brookyn 47. 218. 135,

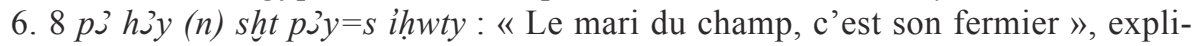
cite probablement un des oracles de la fameuse Chronique démotique, $P$. Bn

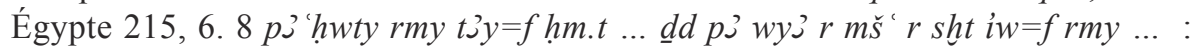

1. S. Sauneron, «L'Abaton de la campagne d'Esna », MDAIK, 16 (1958), p. 275, note a.

2. En B. $\boldsymbol{x} \boldsymbol{\omega} \boldsymbol{x}$, la « tête » ou le « capital » Crum, CD 799a. 
«"Le fermier pleure sa femme ... " C'est-à-dire, le paysan se rendra aux champs en pleurant ....»

Enfin, l'étude d'un autre texte sapiential se référant aux pratiques agricoles, le sixième chapitre de la sagesse d'Aménémopé, nous a permis d'examiner l'intéressante question du bornage des champs et notamment du serment fait sur les limites. Ce serment est effectué par le propriétaire d'une parcelle qui souhaite réaffirmer ses droits sur celle-ci. Pour cela, la personne doit jurer qu'elle détient bien la pièce de terre sur un élément situé à la limite du champ. Il peut s'agir d'une motte de terre ou d'une stèle. Ce fait est avéré sur la longue durée de l'histoire égyptienne. Il est en effet attesté sur certaines peintures du Nouvel Empire comme la tombe de Djeserkarêsoneb à Thèbes ${ }^{1}$ comme dans une archive judiciaire provenant de Crocodilopolis datant du règne de Philopator ${ }^{2}$.

L'an prochain, nous poursuivrons cette étude des figures sociales présentes dans les sagesses avec l'examen de celle du marchand.

1. S. Berger, «A Note on some Scene of Land Measurement », JEA, 30 (1934), p. 54-55, pl. X.

2. A. Helmis, « La terre, les hommes et les dieux : un différent de bornage dans l'Égypte ptolémaïque », dans S. Allam (éd.), Grund und Boden, Tübingen, 1994, p. 327-340. 